\title{
Acute effect and time course of extension and internal rotation stretching of the shoulder on infraspinatus muscle hardness
}

\author{
AUTHOR(S): \\ Kusano, Ken; Nishishita, Satoru; Nakamura, \\ Masatoshi; Tanaka, Hiroki; Umehara, Jun; \\ Ichihashi, Noriaki
}

\section{CITATION:}

Kusano, Ken ...[et al]. Acute effect and time course of extension and internal rotation stretching of the shoulder on infraspinatus muscle hardness. Journal of Shoulder and Elbow Surgery 2017, 26(10): 1782-1788

\section{ISSUE DATE:}

2017-10

URL:

http://hdl.handle.net/2433/243914

\section{RIGHT:}

(C) 2017. This manuscript version is made available under the CC-BY-NC-ND 4.0 license http://creativecommons.org/licenses/by-nc-nd/4.0/.; This is not the published version. Please cite only the published version.; この論文は出版社版でありません。引用の際には 出版社版をご確認ご利用ください。 
1 Acute effect and time course of extension and internal rotation stretching of the shoulder

2 on infraspinatus muscle hardness

3

4 Ken Kusano ${ }^{1)}$, Satoru Nishishita ${ }^{1)}$, Masatoshi Nakamura ${ }^{2)}$, Hiroki Tanaka ${ }^{3)}$, Jun $5 \quad$ Umehara $^{3)}$, Noriaki Ichihashi $^{1)}$

$6 \quad{ }^{1)}$ Human Health Sciences, Graduate School of Medicine, Kyoto University, 53 Shogoin-

$7 \quad$ Kawahara-cho, Kyoto 606-8507, Japan

$8 \quad{ }^{2)}$ Institute for Human Movement and Medical Sciences, Niigata University of Health and

9 Welfare, Japan

$10 \quad{ }^{3)}$ Rehabilitation Unit, Kyoto University Hospital, Kyoto, Japan

*Corresponding author:

13 Ken Kusano $(\bowtie)$

14 Human Health Sciences, Graduate School of Medicine, Kyoto University

1553 Shogoin-Kawahara-cho, Sakyo-ku, Kyoto 606-8507, Japan

16 Telephone: +81-75-751-3935; Fax: +81-75-751-3909

17 E mail: kusano.ken.82u@st.kyoto-u.ac.jp

Acknowledgements

20 We would like to thank Ms. Ibuki and Editage (www.editage.jp) for English language editing. Additionally, this study was supported by the Grant - in - Aid for Scientific Research (B) $15 \mathrm{H} 03043$.

IRB

24 This study has been approved by the Ethics Committee of the Kyoto University 
Effect of static stretching on infraspinatus

25 Graduate School and Faculty of Medicine: No. R0314.

\section{Conflict of Interest}

$27 \quad$ None. 
Effect of static stretching on infraspinatus

28

29

\section{Abstract}

Background: A decrease in flexibility of the infraspinatus muscle causes limitations in the range of shoulder motions. Static stretching (SS) is a useful method to improve muscle flexibility and joint mobility. Previous researchers investigated effective stretching methods for the infraspinatus. However, few researchers investigated the acute effect of SS on the infraspinatus muscle’s flexibility. In addition, the minimum SS time required to increase the infraspinatus muscle's flexibility remains unclear. The aims of this study included investigating the acute effect of SS on the infraspinatus muscle's hardness (an index of muscle flexibility) by measuring shear elastic modulus and determining minimum SS time to decrease the infraspinatus muscle’s hardness.

Methods: This included measuring the effect of SS with extension and internal rotation of the shoulder on the infraspinatus muscle's hardness in 20 healthy men. Hence, shear elastic modulus of the infraspinatus was measured by ultrasonic shear wave elastography before and after every 10 seconds up to 120 seconds of SS.

Finding: Two-way analysis of variance indicated a significant main effect of SS duration on shear elastic modulus. The post hoc test indicated no significant difference between shear elastic modulus after 10 seconds of SS and that before SS. However, shear elastic modulus immediately after a period ranging from 20 seconds to 120 seconds of SS was significantly lower than that before SS.

Interpretation: The results suggested that shoulder extension and internal rotation SS effectively decreased the infraspinatus muscle's hardness. In addition, the results indicated that a period exceeding 20 seconds of SS decreased the infraspinatus muscle's hardness. 
Effect of static stretching on infraspinatus

\section{$51 \quad$ Word count}

$52 \quad 3136$ words

53

$54 \quad$ Keywords

55 Ultrasonic shear wave elastography

$56 \quad$ Shear elastic modulus

$57 \quad$ Infraspinatus

$58 \quad$ Stretching

$59 \quad$ Shoulder

$60 \quad$ Shoulder extension stretching

61 Minimum stretching time 
Effect of static stretching on infraspinatus

\section{Introduction}

Rotator cuff muscles play an important role in shoulder function because these muscles contribute to the dynamic stability of a shoulder joint ${ }^{3}$. Decrease in soft tissue flexibility of the posterior shoulder region, including the infraspinatus, teres minor, and posterior glenohumeral joint capsule, is defined as posterior shoulder tightness ${ }^{4,27}$. Several researchers have indicated that posterior shoulder tightness causes glenohumeral internal rotation deficit (GIRD) and limited range of motion (ROM) in the internal rotation of the shoulder $^{9,22}$. A few others investigated the relationship between shoulder injuries and the manifestations of GIRD ${ }^{8,26}$. The researchers indicated that the manifestations of GIRD are linked to nonspecific shoulder pain ${ }^{8}$, and those affected are at high risk for the development of shoulder pathologic processes ${ }^{26}$. Some reports have shown that treatment of GIRD with physical therapy improves flexibility of the posterior shoulder muscles ${ }^{1,4}$.

In general, static stretching (SS) is recommended as an effective intervention to increase muscle flexibility and joint ROM. Specifically, SS is an effective method that prevents joint contracture, decreases muscle strain, and improves muscle flexibility. Several prior studies have shown that maximum ROM increased immediately after SS ${ }^{5}$, ${ }^{6}$ and that the passive torque or muscle-tendon unit stiffness decreased after SS ${ }^{7,13}$. However, it is not possible to assess the flexibility of individual muscles in the shoulder using these traditional measurements, such as passive torque and muscle stiffness, because of the complex shoulder joint construction and also because of the fact that ROM is affected by pain and stretch tolerance.

Researchers recently assessed muscle hardness as an index of muscle flexibility using ultrasonic shear wave elastography $2,17,29,31$. Ultrasonic shear wave elastography enables reliable measurement of local tissue in vivo. Several authors used shear elastic modulus 
Effect of static stretching on infraspinatus

measured by ultrasonic shear wave elastography and investigated the effect of SS on muscle hardness, ${ }^{18,28}$. In addition, Nakamura et al delineated a significant correlation between rate of change in shear elastic modulus and rate of change in muscle stiffness ${ }^{18}$. Furthermore, Rosskopf et al determined that ultrasonic shear wave elastography is reproducible to assess the shoulder muscles ${ }^{24}$. Therefore, the effect of SS on shoulder muscle hardness could be assessed using ultrasonic shear wave elastography. To stretch muscles effectively, it is important to define appropriate SS positions and a minimum length of time required for SS to increase muscle flexibility. Several researchers suggested SS positions for shoulder muscles based on kinesiology and anatomy ${ }^{16,33}$. A previous cadaveric study showed that the middle portion of the infraspinatus is most stretched in internal rotation with shoulder extension, and the inferior portion of this muscle is most stretched in internal rotation during shoulder elevation as well as during shoulder extension ${ }^{16}$. Therefore, we concluded that shoulder internal rotation with extension or elevation is the effective SS position for the infraspinatus muscle. In addition, the minimum time required for SS to increase infraspinatus muscle flexibility remains unclear. Determination of appropriate SS time could be useful for clinical sites and preparatory activities (exercise and warm-up). A few researchers have examined the minimum time for SS of the lower limbs ${ }^{19,20}$. However, no researchers have examined the minimum time required for SS for upper limb muscles. The SS minimum time required to increase muscle flexibility could differ across the muscles. The aim of this study included investigating the acute effect of SS on infraspinatus muscle hardness and identifying the minimum time required to decrease infraspinatus muscle hardness. 
Effect of static stretching on infraspinatus

\section{Methods}

\subsection{Subjects}

Twenty healthy men with no previous history of orthopedic disease in the shoulder (age, 22.7 \pm 1.5 years) were recruited for this study. Each subject provided written informed consent before taking part in the study. The sample size required for a 2-way analysis of variance (ANOVA) with repeated measures (effect size $=0.25$ [medium], $\alpha$ error $=.05$, and power $=0.95$ ) was calculated using $G^{*}$ power 3.1 software (Heinrich Heine University, Duesseldorf, Germany). We used G* power to calculate the necessary sample size based on the effect size, $\alpha$ error, and power. Elicited results have suggested that 17 subjects were required for the current study.

\subsection{Experimental protocol}

A crossover trial design was adopted to investigate the effect of stretching position on the decrease in muscle hardness. All subjects participated in both the stretching condition and the control condition. A set of the stretching condition consisted of 10 seconds of SS and a 30-second period during which the shear elastic modulus of the infraspinatus muscle was measured. Subjects participated in 12 consecutive sets (total SS time 120 seconds). A set in the control condition involved the subject's relaxing in a prone position for 10 seconds with the subject's upper limbs against the body and a 30-second period to measure shear elastic modulus. Subjects participated in 12 sets. Shear elastic modulus of the infraspinatus was measured before SS (SS0) and 12 times immediately after SS (SS1SS12), thereby corresponding to a total of 13 times in the study.

\subsection{Measurement of the shear elastic modulus}


Effect of static stretching on infraspinatus

132 Ultrasonic shear wave elastography (Aixplorer, SuperSonic Imagine, Axi-en-Provence,

133 France) with a SuperLinear SL 10-2 probe was used to assess the shear elastic modulus

134 of the superior portion of the infraspinatus muscle in the nondominant shoulder. Some

135 subjects had experience in participating in overhead sports. Therefore, we choose the nondominant side to examine only the effect of SS on infraspinatus muscle hardness. The position for measuring the shear elastic modulus of the infraspinatus muscle was termed as the prone position, in which subjects placed their hands behind their backs and brought thumb tips in line with the eighth thoracic vertebra (Fig. 1). The measurement site was defined as the intersection point of 2 lines; 1 line connected a point at the top fourth from the medial margin of spine of the scapula to the inferior angle of the scapula and greater tubercle, and the other line connected the middle point of the spine of the scapula and the inferior angle of the scapula (Fig. 2). These lines were marked on the skin with the subject prone, and the muscle belly was then specifically marked. The probe was placed parallel to the muscle fiber, and it was confirmed that the muscle fiber was uninterrupted on the ultrasonic image.

147 The calculation of the shear elastic modulus was based on previous studies ${ }^{18,21}$. The shear 148 elastic modulus was calculated from the mean shear wave speed within the region of 149 interest. This process was automatically computed by ultrasonic shear wave 150 elastography. All measurements of shear elastic modulus were performed by the same 151 investigator. The shear elastic modulus was measured 3 times. and the mean value was used for the analysis. Each measurement was performed in a period of $<30$ seconds to prevent the effect of measurement position on infraspinatus muscle hardness. Nakamura et al. indicated a significant correlation between rate of change in shear elastic modulus and rate of change in muscle stiffness ${ }^{18}$. 
Effect of static stretching on infraspinatus

2.4 Measurement reliability of the shear elastic modulus

The reliability of the shear elastic modulus measurement was calculated using intraclass correlation (1.1) for the 3 measurements at SS0 in the control condition.

160

\subsection{Measurement of range of $R O M$}

A digital angle gage (WR300; Wixey, Sanibel, FL, USA) was used to measure passive ROM of internal rotation in the shoulder at SS0 and SS12. The ROM measurement of internal rotation was performed with the shoulder at $90^{\circ}$ abduction and the elbow at $90^{\circ}$ flexion at the prone position. Each measurement was performed 3 times, and the mean values were used for analysis. The maximum ROM of internal rotation was defined as the angle at which the inferior angle of the scapula began to move.

\subsection{Stretching position}

170 Extension and internal rotation SS of the shoulder was performed using a previously

171 defined position to stretch the middle and inferior portion of the infraspinatus muscle ${ }^{16}$.

172 The nondominant upper limb was chosen for the SS. The SS starting position was defined as the prone position, in which subjects placed their nondominant hands behind the back

174 with their palms facing upward. In the study, the investigator stabilized the scapula with

1751 hand while cranially moving the subject's nondominant upper limb along the spine (Fig. 3). The stretching was performed to the maximum height that could be achieved by the subject without discomfort or pain. 
Effect of static stretching on infraspinatus

180 Statistical analysis was performed using SPSS (version 18.0; SPSS Japan Inc., Tokyo, 181 Japan). To investigate the effect of SS on infraspinatus muscle hardness and ROM of 182 internal rotation of the shoulder, differences in shear elastic modulus and ROM SS0 and 183 SS1-SS12 were assessed by a 2-way ANOVA with repeated measures using a 2-factor 184 (SS intervention [stretching vs. control] ×measurement duration [13 conditions]) design. 185 The differences of the shear elastic modulus between SS0 and SS1-SS12 were determined 186 using Dunnett post hock test when a significant interactive effect was found. The 187 differences of ROM of internal rotation between SS0 and SS12 were determined using 188 the paired Student t-test. A significance level of .05 was used in all the statistical tests. 
Effect of static stretching on infraspinatus

189

190

191

192

193

194

195

196

197

198

199

200

201

202

203

204

205

206

207

208

209

210

211

\section{Results}

\subsection{Measurement reliability}

The reliability of the shear elastic modulus for the 3 measurements at SS0 in the control condition corresponded to intraclass correlation (1.1) of 0.939 (95\% confidence interval, 0.879-0.973), which was significant.

\subsection{Comparison of shear elastic modulus and ROM}

The shear elastic modulus of each measurement time is shown in Table 1 as a mean \pm standard deviation. The results of the 2-way ANOVA indicated a significant main effect of measurement duration, with significant interaction between SS intervention and measurement duration. The post hoc test indicated that there were no significant differences between SS0 and SS1-SS12 with respect to shear elastic modulus in the control condition. In addition, there was no significant difference between SS0 and SS1 with respect to shear elastic modulus in the stretching condition. However, shear elastic modulus at SS2-SS12 were significantly lower than that at SS0 in the stretching condition.

The ROM of internal rotation at SS0 and SS12 is shown in Table 2 as mean \pm standard deviation values. The results of the 2-way ANOVA indicated a significant main effect of measurement duration with significant interaction between SS intervention and measurement duration. The post hoc test indicated no significant difference between SS0 and SS12 with respect to internal rotation ROM in the control condition. However, the results indicated that internal rotation ROM at SS12 was significantly greater than that at SS0 in the stretching condition. 
Effect of static stretching on infraspinatus

\section{Discussion}

In the study, shear elastic modulus of the infraspinatus muscle was significantly lower and internal rotation ROM was greater after 120 seconds of SS when compared with that before SS. This was the first study to investigate the acute effect of extension and internal rotation SS of the shoulder on the infraspinatus muscle's hardness and ROM by using shear elastic modulus measured via ultrasonic shear wave elastography. Several previous authors examined the effect of SS on decrease in posterior shoulder tightness. Many researchers have used SS with the arm in an elevated position (e.g., the cross-body stretch), in which the shoulder was horizontally adducted ${ }^{14}$ or sleeper stretch, in which the shoulder was internally rotated ${ }^{12}$. However, a previous cadaveric study showed that middle portion of the infraspinatus muscle was most stretched during internal rotation with shoulder extension. In addition, the inferior portion was also stretched in this position and did not exhibit any significant differences from internal rotation in elevation ${ }^{16}$. Hence, the infraspinatus could be stretched more effectively by SS using extension compared with that with respect to SS at elevation.

Previous researchers indicated that ROM of internal rotation and horizontal adduction in the shoulder increased immediately after 60 seconds or 180 seconds of SS ${ }^{12,23}$ and that the infraspinatus muscle’s hardness decreased immediately after 150 seconds of crossbody stretch ${ }^{30}$ or after a 4 week SS intervention ${ }^{34}$. The findings in this study confirmed the effect of extension and internal rotation SS by directly measuring the muscle hardness and indicated that decrease in the infraspinatus muscle's hardness was observed 20 seconds after the initiation of SS. Therefore, it is probably necessary to stretch for at least 20 seconds in order to decrease the infraspinatus muscle’s hardness.

A few publications indicated that the time required for effective SS in the lower limbs 
Effect of static stretching on infraspinatus

is 2.5 minutes ${ }^{20}$ or 7.5 minutes ${ }^{13}$. These time periods are sufficient in decreasing the passive torque and muscle-tendon unit stiffness of the hamstrings. Nevertheless, the infraspinatus muscle's hardness was decreased immediately after 20 seconds of SS in this study. A potential reason for the shorter times of SS in this study compared with that in previous studies could be the differences in muscles structure (size, physiological cross-sectional area [PCSA], and fascicle angles), stabilization of the scapula, and the use of shear elastic modulus as an index of muscle hardness.

The referred researchers examined the effective time needed for SS of the lower limbs ${ }^{13 \text {, }}$ 20. However, in this study, we examined the effective time needed for SS of the infraspinatus muscle. The PCSA of the infraspinatus muscle is very small compared with that of the hamstrings ${ }^{10,11}$. It was hypothesized that muscle tension per PCSA would increase if the PCSA of the muscle decreased and the tension applied by SS was equal. Therefore, the reason for the decrease in the infraspinatus muscle's hardness with respect to shorter SS time could be explained by the large muscle tension per PCSA. In addition, researchers have shown that scapular stabilization during the cross-body stretch increased the effects of stretching on the posterior glenohumeral joint $\mathrm{ROM}^{32}$ and the infraspinatus muscle’s hardness ${ }^{30}$. In this study, the infraspinatus muscle could be effectively stretched because of the stabilization of the scapula during SS. In addition, the shear elastic modulus was used as an index of muscle hardness instead of ROM. In general, the effects of SS were calculated using ROM as an index of joint flexibility. However, several researchers indicated that the assessment of ROM is inadequate in evaluating muscle flexibility because maximum ROM measurements are influenced by pain and stretch tolerance $\mathrm{e}^{15,25}$. In contrast, the shear elastic modulus is calculated using the shear wave propagation speed within the muscle belly. Therefore, the shear elastic modulus assessed 
Effect of static stretching on infraspinatus

260

261

262

263

264

265

266

267

268

269

270

271

272

273

274

275

276

277

278

muscle hardness. In this respect, the shear elastic modulus might be more sensitive to muscle hardness compared with ROM.

The results indicated that there may be differences in the minimum SS time required to decrease muscle hardness among muscles. In the future, it is necessary to investigate the acute effect of SS on the hardness of various muscles and to reveal the relationship between the minimum SS time required to decrease muscle hardness and muscle structures.

This study has a few limitations. First, it was not possible to compare muscle tension during SS in the study with previous literature because of differences in muscle flexibility indices. Second, only the acute effect of SS was examined, and therefore the effect of a long term SS intervention program is unclear. Third, the subjects in this study were healthy young men. Equivalent acute effects of SS cannot be consistently expected in older people and patients with shoulder symptoms. Further research is required to investigate the intervention effect of SS in older people and patients with shoulder symptoms to facilitate clinical application. Fourth, only the nondominant extremity was assessed in this study. So, the effect of SS on the overhead sports player with restriction in ROM of the dominant shoulder is unclear. Fifth, this study protocol was repeated measures. No repeated measure was required to determine if this change was only temporary or maintained. 
Effect of static stretching on infraspinatus

280 The results of this study indicated that the infraspinatus muscle's hardness is decreased

281 whereas ROM of internal rotation is increased immediately after internal rotation and

282 extension SS of the shoulder. Furthermore, the findings suggested that the infraspinatus

283 muscle’s hardness decreased after a minimum of 20 seconds of SS.

284

285

286

287 


\section{References}

290

291

292

293

294

295

296

297

298

299

300

301

302

303

304

305

306

307

308

309

310

311

312

313

314

315

316

317

318

319

320

321

322

1. Bailey LB, Shanley E, Hawkins R, Beattie PF, Fritz S, Kwartowitz D et al. Mechanisms of Shoulder Range of Motion Deficits in Asymptomatic Baseball Players. Am J Sports Med 2015;43:2783-2793. 10.1177/0363546515602446

2. Bercoff J, Tanter M, Muller M, Fink M. The role of viscosity in the impulse diffraction field of elastic waves induced by the acoustic radiation force. IEEE Trans Ultrason Ferroelectr Freq Control 2004;51:1523-1536.

3. Boettcher CE, Cathers I, Ginn KA. The role of shoulder muscles is task specific. J Sci Med Sport 2010;13:651-656. 10.1016/j.jsams.2010.03.008

4. Borsa PA, Wilk KE, Jacobson JA, Scibek JS, Dover GC, Reinold MM et al. Correlation of range of motion and glenohumeral translation in professional baseball pitchers. Am J Sports Med 2005;33:1392-1399. 10.1177/0363546504273490

5. de Weijer VC, Gorniak GC, Shamus E. The effect of static stretch and warm-up exercise on hamstring length over the course of 24 hours. J Orthop Sports Phys Ther 2003;33:727-733. 10.2519/jospt.2003.33.12.727

6. Depino GM, Webright WG, Arnold BL. Duration of maintained hamstring flexibility after cessation of an acute static stretching protocol. J Athl Train 2000;35:56-59.

7. Fowles JR, Sale DG, MacDougall JD. Reduced strength after passive stretch of the human plantarflexors. J Appl Physiol (1985) 2000;89:1179-1188.

8. Giles K, Musa I. A survey of glenohumeral joint rotational range and non-specific shoulder pain in elite cricketers. Phys Ther Sport 2008;9:109-116. 10.1016/j.ptsp.2008.03.002

9. Grossman MG, Tibone JE, McGarry MH, Schneider DJ, Veneziani S, Lee TQ. A cadaveric model of the throwing shoulder: a possible etiology of superior labrum anterior-to-posterior lesions. J Bone Joint Surg Am 2005;87:824-831. 10.2106/JBJS.D.01972

10. Klein Horsman MD, Koopman HF, van der Helm FC, Prosé LP, Veeger HE. Morphological muscle and joint parameters for musculoskeletal modelling of the lower extremity. Clin Biomech (Bristol, Avon) 2007;22:239-247. 10.1016/j.clinbiomech.2006.10.003

11. Langenderfer JE, Patthanacharoenphon C, Carpenter JE, Hughes RE. Variability in isometric force and moment generating capacity of glenohumeral external rotator muscles. Clin Biomech (Bristol, Avon) 2006;21:701-709. 10.1016/j.clinbiomech.2006.02.010 
12. Laudner KG, Sipes RC, Wilson JT. The acute effects of sleeper stretches on shoulder range of motion. J Athl Train 2008;43:359-363. 10.4085/1062-6050-43.4.359

13. Magnusson SP, Simonsen EB, Aagaard P, Kjaer M. Biomechanical responses to repeated stretches in human hamstring muscle in vivo. Am J Sports Med 1996;24:622-628.

14. Manske RC, Meschke M, Porter A, Smith B, Reiman M. A randomized controlled single-blinded comparison of stretching versus stretching and joint mobilization for posterior shoulder tightness measured by internal rotation motion loss. Sports Health 2010;2:94-100. 10.1177/1941738109347775

15. McHugh MP, Kremenic IJ, Fox MB, Gleim GW. The role of mechanical and neural restraints to joint range of motion during passive stretch. Med Sci Sports Exerc 1998;30:928-932.

16. Muraki T, Aoki M, Uchiyama E, Murakami G, Miyamoto S. The effect of arm position on stretching of the supraspinatus, infraspinatus, and posterior portion of deltoid muscles: a cadaveric study. Clin Biomech (Bristol, Avon) 2006;21:474-480. 10.1016/j.clinbiomech.2005.12.014

17. Nakamura M, Hasegawa S, Umegaki H, Nishishita S, Kobayashi T, Fujita K et al. The difference in passive tension applied to the muscles composing the hamstrings Comparison among muscles using ultrasound shear wave elastography. Man Ther 2016;24:1-6. 10.1016/j.math.2016.03.012

18. Nakamura M, Ikezoe T, Kobayashi T, Umegaki H, Takeno Y, Nishishita S et al. Acute effects of static stretching on muscle hardness of the medial gastrocnemius muscle belly in humans: an ultrasonic shear-wave elastography study. Ultrasound Med Biol 2014;40:1991-1997. 10.1016/j.ultrasmedbio.2014.03.024

19. Nakamura M, Ikezoe T, Takeno Y, Ichihashi N. Time course of changes in passive properties of the gastrocnemius muscle-tendon unit during 5 min of static stretching. Man Ther 2013;18:211-215. 10.1016/j.math.2012.09.010

20. Nordez A, Cornu C, McNair P. Acute effects of static stretching on passive stiffness of the hamstring muscles calculated using different mathematical models. Clin Biomech (Bristol, Avon) 2006;21:755-760. 10.1016/j.clinbiomech.2006.03.005

21. Nordez A, Gennisson JL, Casari P, Catheline S, Cornu C. Characterization of muscle belly elastic properties during passive stretching using transient elastography. J Biomech 2008;41:2305-2311. 10.1016/j.jbiomech.2008.03.033

22. Oyama S, Myers JB, Blackburn JT, Colman EC. Changes in infraspinatus crosssectional area and shoulder range of motion with repetitive eccentric external rotator contraction. Clin Biomech (Bristol, Avon) 2011;26:130-135. 
10.1016/j.clinbiomech.2010.09.018

23. Park KN, Kwon OY, Weon JH, Choung SD, Kim SH. Comparison of the effects of local cryotherapy and passive cross-body stretch on extensibility in subjects with posterior shoulder tightness. J Sports Sci Med 2014;13:84-90.

24. Rosskopf AB, Ehrmann C, Buck FM, Gerber C, Flück M, Pfirrmann CW. Quantitative Shear-Wave US Elastography of the Supraspinatus Muscle: Reliability of the Method and Relation to Tendon Integrity and Muscle Quality. Radiology 2016;278:465-474. 10.1148/radiol.2015150908

25. Sale D, Quinlan J, Marsh E, McComas AJ, Belanger AY. Influence of joint position on ankle plantarflexion in humans. J Appl Physiol Respir Environ Exerc Physiol 1982;52:1636-1642.

26. Shanley E, Rauh MJ, Michener LA, Ellenbecker TS, Garrison JC, Thigpen CA. Shoulder range of motion measures as risk factors for shoulder and elbow injuries in high school softball and baseball players. Am J Sports Med 2011;39:1997-2006. $10.1177 / 0363546511408876$

27. Tyler TF, Roy T, Nicholas SJ, Gleim GW. Reliability and validity of a new method of measuring posterior shoulder tightness. J Orthop Sports Phys Ther 1999;29:262269; discussion 270-264. 10.2519/jospt.1999.29.5.262

28. Umegaki H, Ikezoe T, Nakamura M, Nishishita S, Kobayashi T, Fujita K et al. Acute effects of static stretching on the hamstrings using shear elastic modulus determined by ultrasound shear wave elastography: Differences in flexibility between hamstring muscle components. Man Ther 2015;20:610-613. 10.1016/j.math.2015.02.006

29. Umegaki H, Ikezoe T, Nakamura M, Nishishita S, Kobayashi T, Fujita K et al. The effect of hip rotation on shear elastic modulus of the medial and lateral hamstrings during stretching. Man Ther 2015;20:134-137. 10.1016/j.math.2014.07.016

30. Umehara J, Hasegawa S, Nakamura M, Nishishita S, Umegaki H, Tanaka H et al. Effect of scapular stabilization during cross-body stretch on the hardness of infraspinatus, teres minor, and deltoid muscles: An ultrasonic shear wave elastography study. Man Ther 2016. 10.1016/j.math.2016.10.004

31. Umehara J, Ikezoe T, Nishishita S, Nakamura M, Umegaki H, Kobayashi T et al. Effect of hip and knee position on tensor fasciae latae elongation during stretching: An ultrasonic shear wave elastography study. Clin Biomech (Bristol, Avon) 2015;30:1056-1059. 10.1016/j.clinbiomech.2015.09.007

32. Wilk KE, Hooks TR, Macrina LC. The modified sleeper stretch and modified crossbody stretch to increase shoulder internal rotation range of motion in the overhead throwing athlete. J Orthop Sports Phys Ther 2013;43:891-894. 
Effect of static stretching on infraspinatus

395

396

397

398

399

400

401

402

10.2519/jospt.2013.4990

33. Wilk KE, Meister K, Andrews JR. Current concepts in the rehabilitation of the overhead throwing athlete. Am J Sports Med 2002;30:136-151.

34. Yamauchi T, Hasegawa S, Nakamura M, Nishishita S, Yanase K, Fujita K et al. Effects of two stretching methods on shoulder range of motion and muscle stiffness in baseball players with posterior shoulder tightness: a randomized controlled trial. J Shoulder Elbow Surg 2016;25:1395-1403. 10.1016/j.jse.2016.04.025 
Effect of static stretching on infraspinatus

403 Figure 1. The position in which shear elastic modulus of the infraspinatus is measured.

404 One investigator placed the subject's hand behind the subject's back, and brought the tip 405 of the subject's thumb in line with the eighth thoracic vertebra in a prone position. The 406 other investigator measured shear elastic modulus of the infraspinatus with region of 407 interest matching at the center of muscle belly.

408

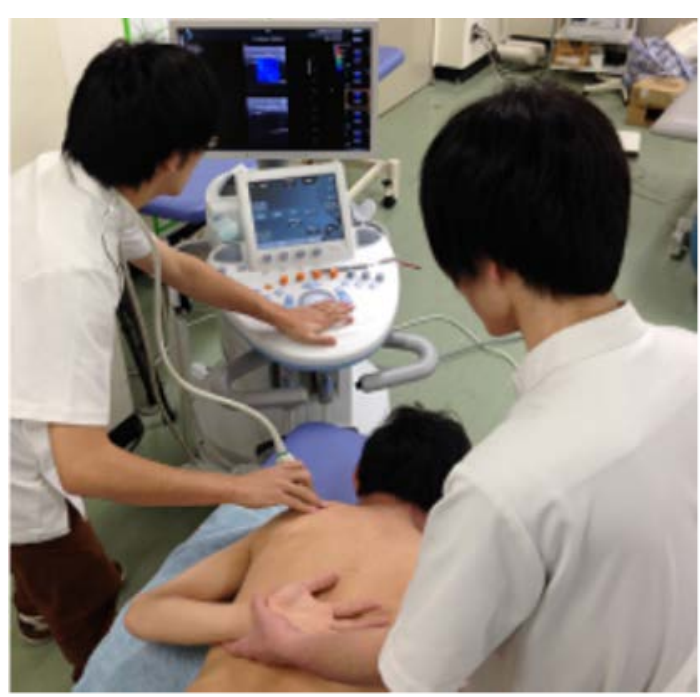


Effect of static stretching on infraspinatus

409 Figure 2. The site of measuring shear elastic modulus of the infraspinatus. The 410 measurement site is defined as the intersection point of 2 lines. One line connects a point 411 at the top fourth from the medial margin of spine of the scapula (MSS) to the inferior 412 angle of the scapula (IA) and greater tubercle (GT). The other line connects the IA and 413 the middle point between MSS and lateral margin of the spine of the scapula (LSS).

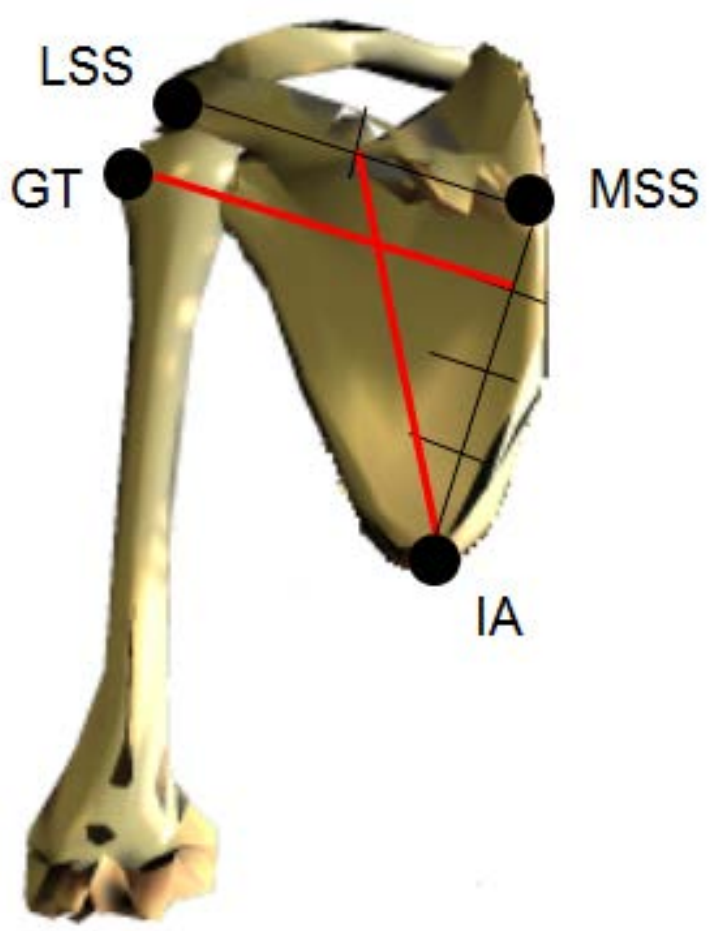


Effect of static stretching on infraspinatus

415 Figure 3. The static stretching position with internal rotation with extension of the

416 shoulder. The nondominant upper limb is selected for the static stretching. The

417 investigator places the subject's nondominant hand behind the subject's back with the

418 subject's palm facing upwards. The investigator stabilizes the subject's scapula with 1

419 hand while moving the nondominant upper limb of the subject cranially with the other

420 hand, such that a point at the maximum possible height on the subject's spine can be

421 reached while ensuring that the back of the subject's hand remains adhered to the

422 subject's back.

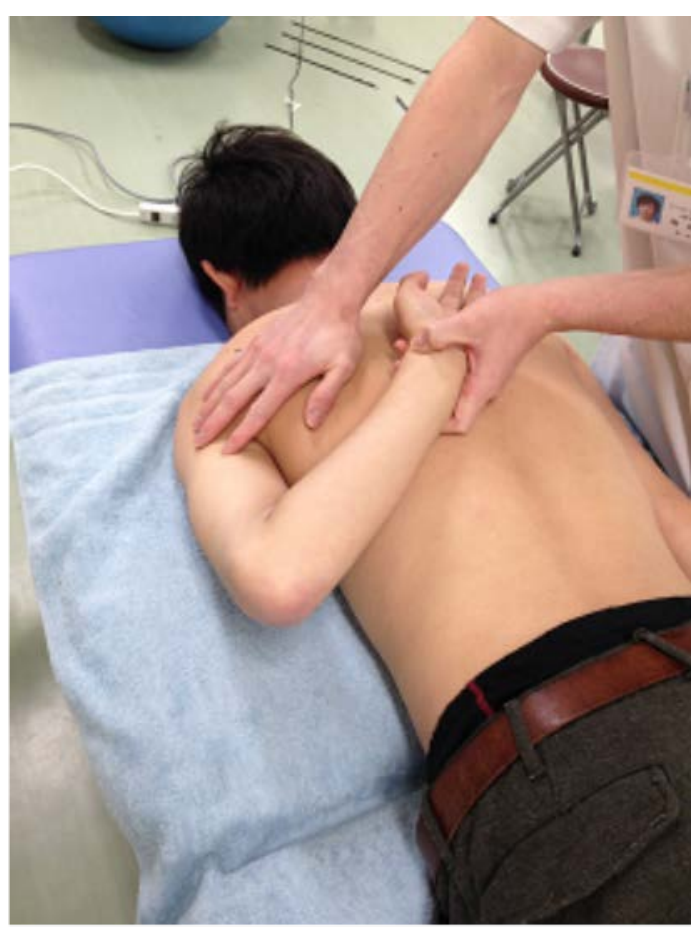


Effect of static stretching on infraspinatus

430 Table 1. Shear elastic modulus of the infraspinatus in the stretching duration from SS0 to $431 \quad$ SS12

\begin{tabular}{|c|c|c|c|c|c|c|}
\hline & \multicolumn{3}{|c|}{ Stretching } & \multicolumn{3}{|c|}{ Control } \\
\hline & Shear elastic & $P$ value & Effect size & Shear elastic & $P$ value & Effect size \\
\hline & Modulus & & & Modulus & & \\
\hline & $(\mathrm{kPa})$ & & & $(\mathrm{kPa})$ & & \\
\hline SS0 & $9.0 \pm 3.2$ & & & $8.9 \pm 3.3$ & & \\
\hline SS1 & $8.2 \pm 3.0$ & .288 & 0.27 & $9.4 \pm 2.9$ & 1.0 & 0.01 \\
\hline SS2 & $7.7 \pm 3.2$ & .012 & 0.43 & $9.2 \pm 3.2$ & 1.0 & 0.05 \\
\hline SS3 & $7.8 \pm 3.0$ & .032 & 0.40 & $9.2 \pm 3.6$ & 1.0 & 0.05 \\
\hline SS4 & $7.5 \pm 2.4$ & .002 & 0.56 & $9.4 \pm 3.6$ & .660 & 0.15 \\
\hline SS5 & $7.9 \pm 2.6$ & .046 & 0.40 & $9.2 \pm 3.1$ & .999 & 0.07 \\
\hline SS6 & $7.4 \pm 2.5$ & .002 & 0.55 & $9.0 \pm 3.0$ & .835 & 0.13 \\
\hline SS7 & $7.9 \pm 2.3$ & .046 & 0.42 & $9.2 \pm 4.0$ & .999 & 0.06 \\
\hline SS8 & $7.4 \pm 2.7$ & .001 & 0.56 & $9.1 \pm 3.3$ & .997 & 0.07 \\
\hline SS9 & $7.6 \pm 3.0$ & .005 & 0.48 & $9.5 \pm 3.2$ & 1.0 & 0.02 \\
\hline SS10 & $7.6 \pm 2.3$ & .006 & 0.52 & $9.5 \pm 3.4$ & 1.0 & 0.03 \\
\hline SS11 & $7.3 \pm 2.6$ & .001 & 0.58 & $9.0 \pm 3.0$ & .927 & 0.11 \\
\hline SS12 & $7.6 \pm 2.5$ & .005 & 0.52 & $9.5 \pm 3.8$ & 1.0 & 0.02 \\
\hline
\end{tabular}

432

433 SS, static stretching.

$434 \quad$ P value and effect size of SS1-SS12 compared with SS0.

435 Values are expressed as mean value \pm standard deviation. The effect size of SS1-SS12

436 with respect to SS0 represents Cohen's d values. 
Effect of static stretching on infraspinatus

437

438 Table 2. Range of motion of internal rotation in the shoulder at $90^{\circ}$ abduction with the 439 elbow in $90^{\circ}$ flexion at SS0 and SS12.

440

\begin{tabular}{ccccccc}
\hline & & Stretching & & & Control & \\
& Range of & P value & Effect size & Range of & P value & Effect size \\
motion & & & motion & & \\
& $\left(^{\circ}\right)$ & & & $\left(^{\circ}\right)$ & & \\
\hline SS0 & $61 \pm 8$ & & & $66 \pm 9$ & & \\
SS12 & $65 \pm 9$ & .001 & 0.52 & $66 \pm 9$ & .287 & 0.08 \\
\hline
\end{tabular}

441

442 SS, static stretching

443 P value and effect size of SS12 compared with SS0.

444 Values are expressed as mean value \pm standard deviation. The effect size of SS12 with 445 respect to SS0 represents Cohen’s d values. 